\title{
Coping Strategies and Quality of Life among Infertile Women in Damanhour City
}

\author{
Nemat Ismail Abdel Aziz Ismail ${ }^{1}$, Amal Awad Abdelnabi Moussa ${ }^{2}$ \\ ${ }^{I}$ Obstetric and Gynecologic Nursing, Faculty of Nursing, Damanhour University, Damanhour, Egypt \\ ${ }^{2}$ Psychiatric Nursing and Mental Health, Faculty of Nursing, Damanhour University, Damanhour, Egypt
}

\begin{abstract}
Infertility and its related problems negatively affect the quality of life. Coping with infertility is important in order to maintain and/or improve the patient's quality of life.

Objective: to assess coping strategies and quality of life among infertile women.

Setting: The study was carried out in the gynecologic outpatient clinic and inpatient department affiliated to National Medical Institution in Damanhour, El-Beheira Governorate.

Subjects: It comprised a convenient sample of 200 women.

Tools: Three tools were utilized for data collection; a structured interview schedule, the COMPI Coping Strategy Scales and Fertility Quality of Life Questionnaire (FertiQoL).

Results: The study revealed that passive-avoidance coping was highly used by $96.5 \%$ of infertile women. Meaning-based coping was highly used by $47.5 \%$ of them. Active-confronting coping was highly used by $37 \%$ of infertile women. While active-avoidance coping was highly used by only $15 \%$ of them. The infertile women had lower total mean score of quality of life 59.375 \pm 15.790 . Total quality of life was found to be positively significantly correlated with active-confronting coping $r=0.205$ (0.004), passive-avoidance coping $r=0.317$ (0.000), and meaning-based coping $r=0.598(0.000)$, while it was found to be negatively significantly correlated with active-avoidance coping $r=-0.435(0.000)$.

Conclusion: the infertile women used passive avoidance coping more frequently than other forms of coping strategies. Moreover, the infertile women obtained lower total mean score of quality of life. In addition, total quality of life was significantly correlated with all the coping strategies.
\end{abstract}

Recommendations: Educational programs should be carried out for nurses to provide them with essential information regarding infertility coping strategies to counseling the infertile women.

Keywords: Infertility, Coping strategies, quality of life

\section{Introduction}

Infertility is a frustrating experience for women. It defined clinically as the failure of a couple to conceive or achieve a pregnancy after one year of regular unprotected sexual intercourse (without any contraception) or demographically as the inability to achieve a live birth. ${ }^{(1,2)}$ There are two types of infertility, primary or secondary. Primary infertility refers to couples who have never conceived a pregnancy, while secondary infertility refers to couples have experienced difficulty conceiving subsequent pregnancies after prior, successful conception. More couples experience secondary infertility than primary infertility. Data indicate that roughly $70 \%$ of women with infertility have had at least one previous pregnancy, and more than one-third of couples experience difficulties conceiving a second child. ${ }^{(3)}$

Epidemiological data indicate that infertility is estimated to involve as many as 186 million people worldwide. It is viewed by experts in the battlefield to be a problem that affects $15 \%$ to $20 \%$ of couples. ${ }^{(4)}$ According to a study conducted by the Egyptian Fertility Care Society and sponsored by the World Health Organization (WHO), infertility in Egypt affects 12 percent of Egyptian couples. Of these women, 4.3 percent suffer from primary infertility and 7.7 percent suffer from secondary infertility. The number of women aged 15 to 49 years exceeds 25 million, which means that at least 3 million women are infertile in Egypt. ${ }^{(5)}$

Infertility is considered a crisis with various biological, psychological, economic, ethical, and cultural consequences. Infertile women will undergo varied psychological distress and in order to overcome psychological distress and maintain their quality of life they need to use appropriate coping strategies. ${ }^{(6)}$ As infertility is an unplanned and unexpected stressor, couples typically lack the knowledge and skill set to adequately manage infertility stress. As a result, couples engage in a variety of coping strategies in an attempt to regain control over their lives and rebalance the disruptions they have experienced in their personal, marital and social relationships. ${ }^{(7)}$ Coping strategies defined as collection of one's cognitive and behavioral efforts which are used to interpret, analyze, and reform a stressful condition, resulting in the reduction of its discomfort ${ }^{(8)}$ Coping has been viewed as a stabilizing factor that may assist individuals in maintaining psychosocial adaptation during stressful events. The process of coping is a very complex response that occurs when an individual attempts to 
remove stress or a perceived threat from the environment. Couples can manage the infertility crisis by using coping strategies during the diagnosis and treatment of infertility. ${ }^{(9)}$

It is important for fertility clinic staff not only to treat the condition of infertility but also to deal with the couples' coping with infertility. Evidence-based knowledge about coping strategies and their consequences is therefore a prerequisite for professional fertility treatment. Fertility clinic staff often needs to advise and support patients in their coping with infertility and treatment related stress. It is therefore important to gain insight into the strategies which influence the patients' coping response. Nurses can provide women education on infertility management, as well as assist with the identification of ineffective coping strategies, and promote effective coping skills and stress management techniques. ${ }^{(10,11)}$

Infertility status and its related factors affect the quality of life through creating psychosocial stress, reduction of life satisfaction, increase of marital conflicts, and decrease of sexual and marital satisfaction. ${ }^{(12,13)}$ Quality of life, according to the WHO is defined as “people's perception of their position in life in the context of the culture and value systems in which they live and in relation to their goals, expectations, standards and concerns". ${ }^{(14)}$ The quality of life concept now makes it possible to assess the effects of treatment on health conditions, comprehending outcomes beyond symptomatology, morbidity, and mortality. Moreover, it emphasizes the patients' reported outcomes since the patients are considered to be most capable of measuring their own condition subjectively in several areas of their lives. ${ }^{(15)}$ Clinicians tend to measure the results of interventions through the decrease in symptoms and the involution of the disease. Patients do so through the feeling of comfort or the ability to resume daily activities satisfactorily. ${ }^{(16)}$

The importance of quality of life as one of the aspects of health has attracted the attention of many researchers. Quality of life has become one of the important issues today and is seen as one of the measurable criteria for evaluation of treatment. It is also related to life satisfaction, self-image, health factors, and functional, social, economic and cultural matters; as mentioned earlier, infertility is one of the issues that can affect every aspect of quality of life. ${ }^{(17,18)}$

Evaluation of quality of life helps establish an effective relationship between a patient and his/her treatment team, i.e. physicians and nurses. This increases patients' knowledge about their diseases and diagnosis, the advantages and disadvantages of different treatments and helps infertile individuals make informed decisions about treatment methods. Nurses can improve patients' quality of life via nursing processes: they should make careful evaluations of patients' quality of life and regulate their care models accordingly. Evaluation of quality of life of infertile couples helps health care providers and special-care nurses choose the right treatment and support care. Improvement of infertile couples' quality of life can create happy families and a stable society. $(19,20)$

As the way women cope with infertility can be modified by providing appropriate information, nurses can assess the coping strategies used by women and educate to promote the use of appropriate coping strategies to enhance quality of life. Therefore, this study aimed to assess coping strategies and quality of life among infertile women.

\section{The aim of the study:}

This study aimed to assess coping strategies and quality of life among infertile women in Damanhour city.

\section{Research questions:}

1. What are the coping strategies of infertile women in Damanhour city?

2. What is the quality of life of infertile women Damanhour city?

\section{Materials And Method:}

Materials

Design: An exploratory descriptive design was used in this study.

Setting: The study was conducted in gynecologic outpatient clinic and inpatient department affiliated to National Medical Institution in Damanhour, El-Beheira Governorate. This hospital was selected because it is the main referral hospital.

\section{Subjects:}

It comprised a convenient sample of 200 women who was available during data collection in the previously mentioned setting. They were selected according to the following criteria: Have primary or secondary infertility, had no chronic illness and no diagnosed psychiatric illness and willing to participate in the study. The sample size was determined based on Epi -Info 7 program, using 5\% acceptable error, $95 \%$ confidence coefficient

Tools: Three tools were utilized for data collection.

Tool (I): A socio-demographic and reproductive history sheet:

A structured interview schedule was developed and utilized by the researchers for data collection; it entailed data related to socio-demographic characteristics such as age, education, occupation, residence, type of family, 
monthly income, age at marriage and duration of marriage. It also comprised data related to infertility such as type (primary or secondary), duration and causes.

\section{Tool (II): The COMPI Coping Strategy Scales:}

A modified version of COMPI coping strategy scale, used for evaluating the coping with infertility problems of infertile couples, it was developed by Schmidt et al (2005). ${ }^{(21)}$ It was translated into Arabic by specialist in English language translation. The version was revised and then back translated into English by another translator. The translation was refined after back translation until agreement was obtained among the two translators. The COMPI Coping Strategy Scale is a 19 item scale with four subscales. It can be applied to both women and men. Active-avoidance coping (e.g. I avoid being with pregnant women or children) was measured by four items. Active-confronting coping (e.g. I ask other childless people for advice) was measured by seven items. Passive-avoidance coping (e.g. I try to forget everything about our childlessness) was measured by three items and meaning-based coping (e.g. I find other life goals) was measured by five items. The COMPI Coping Strategy Scale is a four-point Likert scale ranging from 0 for not use, 1 for low use, 2 for moderate use and 3 for high use. The total score of Active-avoidance subscale ranged from zero to 12, it was classified as follows: from 0 to $<4$ were considered low use, from 4 to $<8$ were considered moderate use and from 8 to 12 were considered high use. The total score of active-confronting subscale ranged from zero to 21 , it was classified as follows: from 0 to $<7$ were considered low use, from 7 to $<14$ were considered moderate use and from 14 to 21 were considered high use. The total score of passive-avoidance subscale ranged from zero to 9 , it was classified as follows: from 0 to $<3$ were considered low use, from 3 to $<6$ were considered moderate use and from 6 to 9 were considered high use. The total score of meaning-based coping ranged from zero to 15, it was classified as follows: from 0 to $<5$ were considered low use, from 5 to $<10$ were considered moderate use and from 10 to 15 were considered high use. ${ }^{(22)}$

\section{Tool (III): Fertility Quality of Life Questionnaire (FertiQoL):}

The FertiQoL questionnaire was developed by international group of institutions (the European Society of Human Reproduction and Embryology, the American Society of Reproductive Medicine and Merck, Geneva, Switzerland) in 2011. ${ }^{(23)}$ It is a self-report international questionnaire. It is specifically designed for infertile patients to assess their quality of life. The FertiQoL was produced in English and translated into 20 languages, including Arabic, the Arabic version of the FertiQoL questionnaire was used in the present study. The FertiQoL questionnaire consists of two parts: the Core and the Treatment parts. The Core-FertiQoL part contains 24 questions categorized into four subscales, including the Emotional, Mind/Body, Relational, and Social subscales. The Emotional subscale score shows the impact negative emotions (e.g., jealousy \& resentment, sadness, depression) have on quality of life. The Mind-Body subscale score shows the impact infertility has had on physical health (e.g., fatigue, pain) cognition (e.g., concentration) and behavior (e.g., disrupted daily activities, delayed life plans). The Relational subscale score shows the impact fertility problems have had on the components (e.g., sexuality, communication, commitment) of marital relationship. The Social subscale score shows the extent to which social interactions have been affected by fertility problems (e.g., social inclusion, expectations, stigma, and support). The treatment-FertiQoL part contains 10 questions categorized into two subscales including the Treatment Environment and Treatment Tolerability subscales. The Treatment Environment subscale score shows the extent to which the accessibility and quality of the treatment has impacted the quality of life. The Treatment Tolerability subscale score shows the extent to which infertile woman have experienced mental and physical symptoms as a result of the fertility treatment and the impact this has had on daily life. Items from these subscales are presented in the questionnaire randomly and rated on a response scale of 0 to 4 . The subscales and total FertiQoL scores are computed and transformed to achieve a range of 0 to 100 , where higher scores indicate better quality of life. ${ }^{(24)}$

\section{Method}

1. Approval of the responsible authorities was obtained through official letter from the Faculty of Nursing Damanhour University to the director of National Medical Institution in Damanhour, El-Beheira Governorate to collect the necessary data.

2. After reviewing of the relevant literature, tool I was developed by the researchers, tool II was adapted and translated into Arabic language and tool III (FertiQoL Arabic version) was adopted. The tools (I, II, III) were validated by juries of five experts in the obstetric and gynecologic nursing as well as psychiatric nursing. The reliability of the tool II and tool III were tested by test- retest technique the Cronbach's alpha test for tool II and tool III was $0.88 \& 0.85$ respectively.

3. A pilot study was carried out on 20 infertile women, excluded from the study to ascertain the relevance, clarity and applicability of the tools as well as estimate the time needed to complete the tools. 
4. The final form of the tools was used by the researchers to collect data from the study subjects. Women attending gynecologic outpatient clinic were interviewed before examination, while women in inpatient department were interviewed during their stay in the hospital. The time taken to complete the study tools ranged from 10 to 15 minutes for each woman. The data collection consumed 6 months, from the beginning of February 2016 till the end of July 2016.

\section{Ethical considerations:}

Informed oral consent was obtained from all women after providing an appropriate explanation about the purpose of the study and nature of the research. The confidentiality and anonymity of individual responses, volunteer participation and right to refuse participating in the study were emphasized to the women.

\section{Statistical analysis:}

The collected data were coded, tabulated and analyzed using Statistical Package for Social Sciences (SPSS) version 16. The percentage, mean, standard deviation, Chi-square-test, and Fisher Exact-test were used to summarize data. The comparison between mean scores was performed using t-test and ANOVA test. Significance was adopted at $\mathrm{p}<0.05$ for interpretation of results of tests of significance, all P-values are twosided.

\section{Results}

Table (1) indicates the distribution of studied infertile women according to their socio-demographic characteristics. It was observed that the mean age of women was $28.96 \pm 6.85$ years. More than one-third $(36 \%)$ of women were illiterate or just read \& write, while $30 \%$ of them had secondary education. The majority of women $(90 \%)$ were housewives. More than three-fifths $(64 \%)$ of them lived in rural areas. In addition, more than one-half (56.5\%) of women had nuclear family. Slightly more than two-thirds $68 \%$ of them had enough monthly income. More than one-half (54\%) of women married at the age of 20 to less than 30 years with the mean age at marriage $22.30 \pm 5.38$. the majority $76 \%$ of women has duration of marriage from one to less than 10 years, with the mean duration of marriage $6.44 \pm 4.80$.

Table (2) reveals distribution of studied infertile women according to their infertility history. It was revealed that more than two-thirds $(68 \%)$ of women had primary infertility, while slightly less than one-third (32\%) of them had secondary infertility. More than two-fifths (42\%) of women were infertile for 1 to less than 3 years, while $35 \%$ of them were infertile for 3 to less than 6 years and $23 \%$ were infertile for 6 years or more. The cause of infertility was $67 \%$ female factor and $16 \%$ male factor.

Table (3) illustrates distribution of studied infertile women according to their coping strategy. Activeavoidance coping was moderate used by $44 \%$ of women, and low used by $41 \%$ of them. The mean use of activeavoidance coping was $4.530 \pm 2.720$. While active-confronting coping was moderate used by $42.5 \%$ of women and highly used by $37 \%$ of them. The mean use of active-confronting coping was 11.510 \pm 5.094 . Passiveavoidance coping was highly used by $96.5 \%$ of women, while it was moderate used by $3.5 \%$ of them. The mean use of passive-avoidance coping was $7.895 \pm 1.301$. Meaning-based coping was highly used by $47.5 \%$ of women, and moderate used by $46 \%$ of them. The mean use of meaning-based coping was $9.665 \pm 3.331$.

Table (4) presents distribution of studied infertile women according to their quality of life based on FertiQoL scores. Regarding core FertiQoL part, the mean emotional subscale score was $58.833 \pm 23.210$, and the mean mind/body subscale score was $61.799 \pm 20.904$. While the mean relational subscale score was $69.779 \pm 20.994$, and the mean social subscale score was $66.712 \pm 18.836$. Concerning treatment FertiQoL part, the mean environment subscale score was $41.209 \pm 15.085$, and the mean tolerability subscale score was $56.489 \pm 25.042$. The total mean quality of life (total FertiQoL) was $59.375 \pm 15.790$.

Table (5) explains the relationship between studied infertile women's socio-demographic characteristics and their coping strategies. Concerning active-avoidance coping, the women have primary or preparatory education have high mean score of active-avoidance coping than other levels of education. The relationship was found to be statistically significant, where $\mathrm{P}=0.000$. The women married at the age 20 to less than 30 years have high score of active-avoidance coping more than women married at others ages, where there was statistically significant difference $\mathrm{P}=0.033$. On the other hand, no statistically significant differences were found between age, occupation, current residence, type of family, family income, duration of marriage and active-avoidance coping. In relation to active-confronting coping, the women have university education have high mean score of active-confronting coping than other levels of education, where there was statistically significant difference $\mathrm{P}=0.014$. The women married at age 30 years or more have high score of active-confronting coping more than women married at others ages, where there was statistically significant difference $\mathrm{P}=0.006$. On the other hand, no statistically significant differences were found between age, occupation, current residence, type of family, family income, duration of marriage and active-confronting coping. Concerning passive-avoidance coping, there was statistically significant difference between age, level of education, occupation, residence, age at marriage 
and passive-avoidance coping $(\mathrm{P}=0.001, \mathrm{P}=0.001, \mathrm{P}=0.022, \mathrm{P}=0.003 \& \mathrm{P}=0.000$ respectively). While no statistically significant differences were found between type of family, family income, duration of marriage and passive-avoidance coping. Regarding meaning-based coping, there was statistically significant difference between age, level of education, occupation, residence, age at marriage, duration of marriage and meaningbased coping $(\mathrm{P}=0.000, \mathrm{P}=0.000, \mathrm{P}=0.000, \mathrm{P}=0.000, \mathrm{P}=0.000 \& \mathrm{P}=0.004$ respectively). While no statistically significant differences were found between type of family, family income and meaning-based coping.

Table (6) shows relationship between studied infertile women's infertility history and their coping strategies. Regarding type of infertility, the women with primary infertility have significantly higher mean of using active avoidance coping $(4.7941 \pm 2.883)$ than women with secondary infertility $(3.969 \pm 2.254)$. On the other hand, the women with secondary infertility have higher means of using active-confronting, passiveavoidance and meaning-based coping $(12.578 \pm 4.234,8.453 \pm 0.890 \& 10.953 \pm 3.083$ respectively) compared to the women with primary infertility $(11.007 \pm 5.393,7.632 \pm 1.381 \& 9.059 \pm 3.281$ respectively). There was a statistically significant difference between active avoidance, active-confronting, passive-avoidance, meaningbased coping and type of infertility, where $(\mathrm{P}=0.045, \mathrm{P}=0.042, \mathrm{P}=0.000 \& \mathrm{P}=0.000$ respectively). In relation to duration of infertility, the women with duration of infertility 6 years or more have higher mean of using passive avoidance coping $(8.467 \pm 0.894)$ than the women with other duration $(7.435 \pm 1.340 \& 8.086 \pm 1.294)$. While the women with duration of infertility 3 to less than 6 years have higher mean of using meaning-based coping $(10.486 \pm 3.434)$ than the women with other duration $(9.259 \pm 3.259 \& 9.156 \pm 3.126)$. There was a statistically significant difference between passive-avoidance, meaning-based coping and duration of infertility, where $(\mathrm{P}=0.000 \& \mathrm{P}=0.037$ respectively). Considering cause of infertility, the women with unexplained cause of infertility have higher mean of using active avoidance coping (7.143 \pm 2.673$)$ than the women with other cause of infertility. On the other hand, the women with infertility caused by male and female factors have higher mean of using active-confronting, passive-avoidance and meaning-based coping (13.923 $\pm 4.931,8.885 \pm 0.326$ \& $11.923 \pm 2.607$ respectively) than the women with other infertility cause. There was a statistically significant difference between active-avoidance, active-confronting, passive-avoidance, meaning-based coping and the cause of infertility, where $(\mathrm{P}=0.023, \mathrm{P}=0.048, \mathrm{P}=0.000 \& \mathrm{P}=0.000$ respectively).

Table (7) explains relationship between studied infertile women's socio-demographic characteristics and their total score of quality of life. The younger women aged less than 20 years have significantly lower mean of total score of quality of life than the older women $(\mathrm{P}=0.030)$. The women with University education have significantly higher mean of quality of life than the women with other level of education $(P=0.000)$. The working women have significantly higher mean quality of life $67.770 \pm 16.602$ than housewives women 58.442 \pm $15.465(\mathrm{P}=0.025)$. The women lived in urban areas have significantly higher mean of quality of life $65.186 \pm$ 13.522 than the women lived in rural areas 56.035 16.077 , where $\mathrm{P}=0.000$. In addition, the women with enough family income and can save from it have significantly higher mean quality of life $(\mathrm{P}=0.002)$. On the other hand, type of family, age at marriage and duration of marriage have no statistically significant difference.

Table (8) shows relationship between studied infertile women's infertility history and their total score of quality of life. The women with secondary infertility obtained significantly higher mean score of quality of life $63.966 \pm 16.049$ than the women with primary infertility $57.215 \pm 15.252$, where $(\mathrm{P}=0.006)$. and the women with infertility caused by male factor obtained significantly higher mean score of quality of life than the women with other cause of infertility, where $(\mathrm{P}=0.000)$. On the other hand, the duration of infertility has no statistically significant difference.

Table (9): presents correlation between coping strategies and total score of quality of life of studied infertile women. Active-avoidance coping was found to be negatively correlated with active-confronting coping, passive-avoidance coping, meaning-based coping and total quality of life $(r=-0.524, r=-0.180, r=-0.168 \& r=-$ 0.435 respectively). Active-confronting coping was found to be positively correlated with passive-avoidance coping and total quality of life $(r=0.198 \& \mathrm{r}=0.205)$. Passive-avoidance coping was found to be positively correlated with meaning-based coping and total quality of life $(r=0.320 \& r=0.317)$. Meaning-based coping was found to be positively correlated with total quality of life ( $r=0.598)$. Total quality of life was found to be positively correlated with active-confronting, passive-avoidance and meaning-based coping, while it was found to be negatively correlated with Active-avoidance coping. Total quality of life was significantly correlated with all the coping strategies. 
Table (1) Distribution of studied infertile women according to their socio-demographic characteristics

\begin{tabular}{|c|c|c|}
\hline Socio-demographic characteristics & $\mathrm{N}=\mathbf{2 0 0}$ & $\%$ \\
\hline $\begin{array}{l}\text { Age (years): } \\
<20 \\
20-30 \\
30-40 \\
40 \text { or more } \\
\end{array}$ & $\begin{array}{l}20 \\
89 \\
70 \\
21 \\
\end{array}$ & $\begin{array}{l}10.0 \\
44.5 \\
35.0 \\
10.5\end{array}$ \\
\hline Mean \& SD & \multicolumn{2}{|c|}{$28.96 \pm 6.85$} \\
\hline $\begin{array}{l}\text { Level of education: } \\
\text { Illiterate/read \& write } \\
\text { Primary/ preparatory } \\
\text { Secondary } \\
\text { University }\end{array}$ & $\begin{array}{l}72 \\
42 \\
60 \\
26\end{array}$ & $\begin{array}{l}36 \\
21 \\
30 \\
13 \\
\end{array}$ \\
\hline $\begin{array}{l}\text { Occupation: } \\
\text { Housewife } \\
\text { Working } \\
\end{array}$ & $\begin{array}{l}180 \\
20 \\
\end{array}$ & $\begin{array}{l}90 \\
10 \\
\end{array}$ \\
\hline $\begin{array}{l}\text { Current residence: } \\
\text { Rural } \\
\text { Urban } \\
\end{array}$ & $\begin{array}{l}128 \\
72 \\
\end{array}$ & $\begin{array}{l}64 \\
36 \\
\end{array}$ \\
\hline $\begin{array}{l}\text { Type of family: } \\
\text { Nuclear } \\
\text { Extended } \\
\end{array}$ & $\begin{array}{l}113 \\
87 \\
\end{array}$ & $\begin{array}{r}56.5 \\
43.5 \\
\end{array}$ \\
\hline $\begin{array}{l}\text { Family income: } \\
\text { Enough \&can save from it } \\
\text { Only enough } \\
\text { Not enough } \\
\end{array}$ & $\begin{array}{l}6 \\
136 \\
58 \\
\end{array}$ & $\begin{array}{l}3 \\
68 \\
29 \\
\end{array}$ \\
\hline $\begin{array}{l}\text { Age at marriage (years): } \\
<20 \\
20-30 \\
30 \text { or more }\end{array}$ & $\begin{array}{l}67 \\
108 \\
25\end{array}$ & $\begin{array}{l}33.5 \\
54.0 \\
12.5\end{array}$ \\
\hline Mean \& SD & \multicolumn{2}{|c|}{$22.30 \pm 5.38$} \\
\hline $\begin{array}{l}\text { Duration of marriage (years): } \\
1-10 \\
10 \text { or more }\end{array}$ & $\begin{array}{l}152 \\
48 \\
\end{array}$ & $\begin{array}{l}76.0 \\
24.0 \\
\end{array}$ \\
\hline Mean \& SD & \multicolumn{2}{|c|}{$6.44 \pm 4.80$} \\
\hline
\end{tabular}

Table (2) Distribution of women according to their infertility history

\begin{tabular}{|l|l|l|}
\hline Infertility history & $\mathbf{N = 2 0 0}$ & \% \\
\hline Type of infertility: & 136 & \\
- Primary infertility & 64 & 68.0 \\
- Secondary infertility & & 32.0 \\
\hline Duration of infertility (years): & 84 & \\
1 - 3 & 70 & 42.0 \\
$3-6$ & 46 & 35.0 \\
6 or more & & 23.0 \\
\hline Cause of Infertility & 134 & \\
- Female factor & 32 & 67.0 \\
- Male factor & 27 & 16.0 \\
- Both (male \& female) & 7 & 13.5 \\
- Unexplained & 03.5 \\
\hline
\end{tabular}


Table (3) Distribution of studied infertile women according to their coping strategy based on COMPI coping strategy scales

\begin{tabular}{|l|l|l|}
\hline The COMPI coping strategy scales & $\mathbf{N = 2 0 0}$ & \% \\
\hline Active-avoidance coping & \multicolumn{2}{|l|}{} \\
- Low use (from 0 to less than 4) & 82 & 41.0 \\
-Moderate use (from 4 to less than 8) & 88 & 44.0 \\
-High use (from 8 to 12) & 30 & 15.0 \\
\hline Mean \& SD & $4.530 \pm 2.720$ & \\
\hline Active-confronting coping & & 20.5 \\
-Low use (from 0 to less than 7) & 41 & 42.5 \\
-Moderate use (from 7 to less than 14) & 85 & 37.0 \\
- High use (from 14 to 21) & 74 & \\
\hline Mean \& SD & $11.510 \pm 5.094$ & 0.00 \\
\hline Passive-avoidance coping & & 3.5 \\
- Low use (from 0 to less than 3) & 0 & 96.5 \\
- Moderate use (from 3 to less than 6) & 7 & 193 \\
- High use (from 6 to 9) & $7.895 \pm 1.301$ & \\
\hline Mean \& SD & & 6.5 \\
\hline Meaning-based coping & 13 & 46.0 \\
- Low use (from 0 to less than 5) & 92 & 47.5 \\
\hline - Moderate use (from 5 to less than 10) & 95 & \\
\hline - High use (from 10 to 15) & $9.665 \pm 3.331$ & \\
\hline Mean \& SD & & \\
\hline
\end{tabular}

Table (4) Distribution of studied infertile women according to their quality of life based on FertiQoL scores

\begin{tabular}{|l|l|}
\hline Quality of life & Mean \& SD \\
\hline Core FertiQoL: & \\
- Emotional & $58.833 \pm 23.210$ \\
- Mind/body & $61.799 \pm 20.904$ \\
- Relational & $69.779 \pm 20.994$ \\
- Social & $66.712 \pm 18.836$ \\
\hline Treatment FertiQoL: & \\
- Environment & $41.209 \pm 15.085$ \\
- Tolerability & $56.489 \pm 25.042$ \\
\hline Total quality of life (FertiQoL) & $59.375 \pm 15.790$ \\
\hline
\end{tabular}


Table (5) Relationship between studied infertile women's socio-demographic characteristics and their coping strategies

\begin{tabular}{|c|c|c|c|c|}
\hline \multirow[t]{2}{*}{$\begin{array}{l}\text { Socio-demographic } \\
\text { characteristics }\end{array}$} & $\begin{array}{l}\text { Active-avoidance } \\
\text { coping }\end{array}$ & $\begin{array}{l}\text { Active- } \\
\text { confronting } \\
\text { coping }\end{array}$ & $\begin{array}{l}\text { Passive-avoidance } \\
\text { coping }\end{array}$ & $\begin{array}{l}\text { Meaning-based } \\
\text { coping }\end{array}$ \\
\hline & Mean \& SD & Mean \& SD & Mean \& SD & Mean \& SD \\
\hline \multicolumn{5}{|l|}{ Age (years): } \\
\hline$<20$ & $3.900 \pm 2.990$ & $10.550 \pm 5.951$ & $7.000 \pm 0.858$ & $7.700 \pm 2.386$ \\
\hline 20- 30 & $4.629 \pm 2.838$ & $11.573 \pm 4.880$ & $7.787 \pm 1.519$ & $10.101 \pm 3.012$ \\
\hline $30-40$ & $4.400 \pm 2.293$ & $11.686 \pm 5.038$ & $8.257 \pm 0.912$ & $10.514 \pm 3.513$ \\
\hline 40 or more & $5.143 \pm 3.260$ & $11.571 \pm 5.582$ & $8.000 \pm 1.342$ & $6.857 \pm 2.651$ \\
\hline $\mathbf{F}(\mathbf{P})$ & $0.804(0.493)$ & $0.267(0.849)$ & $5.575(0.001)$ & $10.676(0.000)$ \\
\hline \multicolumn{5}{|l|}{ Level of education: } \\
\hline Illiterate/read \& write & $4.923 \pm 2.226$ & $8.689 \pm 3.807$ & $8.116 \pm 1.345$ & $9.246 \pm 3.283$ \\
\hline Primary/ preparatory & $6.089 \pm 3.059$ & $11.385 \pm 4.262$ & $8.539 \pm 0.859$ & $8.000 \pm 2.558$ \\
\hline Secondary & $3.971 \pm 2.717$ & $12.507 \pm 5.522$ & $7.617 \pm 1.166$ & $9.500 \pm 2.955$ \\
\hline University & $3.833 \pm 2.141$ & $12.533 \pm 5.057$ & $7.556 \pm 1.439$ & $14.039 \pm 1.216$ \\
\hline $\mathbf{F}(\mathbf{P})$ & $6.897(0.000)$ & $3.628(0.014)$ & $6.077(0.001)$ & $26.411(0.000)$ \\
\hline \multicolumn{5}{|l|}{ Occupation: } \\
\hline Housewife & $4.406 \pm 2.715$ & $11.628 \pm 5.245$ & $7.839 \pm 1.325$ & $9.294 \pm 3.206$ \\
\hline Working & $5.650 \pm 2.560$ & $10.450 \pm 3.364$ & $8.400 \pm .940$ & $13.000 \pm 2.534$ \\
\hline $\mathbf{T}(\mathbf{P})$ & $2.049(0.052)$ & $0.981(0.328)$ & $2.415(0.022)$ & $6.026(0.000)$ \\
\hline \multicolumn{5}{|l|}{ Current residence: } \\
\hline Rural & $4.472 \pm 2.788$ & $11.441 \pm 5.351$ & $7.701 \pm 1.353$ & $8.906 \pm 3.203$ \\
\hline Urban & $4.630 \pm 2.611$ & $11.630 \pm 4.647$ & $8.233 \pm 1.137$ & $10.986 \pm 3.151$ \\
\hline $\mathbf{T}(\mathbf{P})$ & $0.394(0.694)$ & $0.252(0.801)$ & $2.969(0.003)$ & $4.469(0.000)$ \\
\hline \multicolumn{5}{|l|}{ Type of family: } \\
\hline Nuclear & $4.759 \pm 3.068$ & $11.071 \pm 5.067$ & $8.054 \pm 1.214$ & $9.991 \pm 3.240$ \\
\hline Extended & $4.239 \pm 2.181$ & $12.068 \pm 5.103$ & $7.693 \pm 1.384$ & $9.250 \pm 3.418$ \\
\hline $\mathbf{T}(\mathbf{P})$ & $1.343(0.180)$ & $1.375(0.171)$ & $1.928(0.055)$ & $1.557(0.121)$ \\
\hline \multicolumn{5}{|l|}{ Family income: } \\
\hline Enough \&can save from it & $6.000 \pm 4.382$ & $13.500 \pm 8.216$ & $9.000 \pm 0.000$ & $11.000 \pm 3.286$ \\
\hline Only enough & $4.504 \pm 2.679$ & $11.183 \pm 5.120$ & $7.861 \pm 1.220$ & $9.891 \pm 3.026$ \\
\hline Not enough & $4.439 \pm 2.626$ & $12.088 \pm 4.638$ & $7.860 \pm 1.505$ & $8.983 \pm 3.930$ \\
\hline $\mathbf{F}(\mathbf{P})$ & $0.914(0.402)$ & $1.109(0.332)$ & $2.260(0.107)$ & $2.012(0.136)$ \\
\hline \multicolumn{5}{|l|}{ Age at marriage (years): } \\
\hline$<20$ & $4.567 \pm 3.021$ & $10.254 \pm 5.264$ & $7.328 \pm 1.561$ & $8.776 \pm 2.833$ \\
\hline $20-30$ & $4.806 \pm 2.470$ & $11.713 \pm 5.044$ & $8.194 \pm 1.045$ & $10.583 \pm 3.262$ \\
\hline 30 or more & $3.240 \pm 2.634$ & $14.000 \pm 3.808$ & $8.120 \pm 1.054$ & $8.080 \pm 3.730$ \\
\hline $\mathrm{F}(\mathbf{P})$ & $3.456(0.033)$ & $5.332(0.006)$ & $10.507(0.000)$ & $10.177(0.000)$ \\
\hline \multicolumn{5}{|l|}{$\begin{array}{l}\text { Duration of marriage } \\
\text { (years): }\end{array}$} \\
\hline $1-10$ & $4.487 \pm 2.728$ & $11.724 \pm 5.022$ & $7.803 \pm 1.391$ & $10.046 \pm 3.411$ \\
\hline $10-20$ & $4.667 \pm 2.716$ & $10.833 \pm 5.313$ & $8.188 \pm .915$ & $8.458 \pm 2.767$ \\
\hline $\mathbf{T}(\mathbf{P})$ & $0.399(0.691)$ & $1.025(0.308)$ & $1.797(0.074)$ & $2.933(0.004)$ \\
\hline
\end{tabular}

$\mathrm{F}(\mathrm{P})$ : ANOVA- test \& P for ANOVA test $\quad \mathrm{T}(\mathrm{P})$ : T-test \& P for T-test

*: Significant at $\mathrm{P} \leq 0.05$

Table (6) Relationship between studied infertile women's infertility history and their coping strategies

\begin{tabular}{|l|l|l|l|l|}
\hline \multirow{2}{*}{ Infertility history } & $\begin{array}{l}\text { Active-avoidance } \\
\text { coping }\end{array}$ & $\begin{array}{l}\text { Active-confronting } \\
\text { coping }\end{array}$ & $\begin{array}{l}\text { Passive-avoidance } \\
\text { coping }\end{array}$ & $\begin{array}{l}\text { Meaning-based } \\
\text { coping }\end{array}$ \\
\cline { 2 - 5 } & Mean \& SD & Mean \& SD & Mean \& SD & Mean \& SD \\
\hline Type of infertility: & & & & \\
- Primary infertility & $4.7941 \pm 2.883$ & $11.007 \pm 5.393$ & $7.632 \pm 1.381$ & $9.059 \pm 3.281$ \\
- Secondary infertility & $3.969 \pm 2.254$ & $12.578 \pm 4.234$ & $8.453 \pm 0.890$ & $10.953 \pm 3.083$ \\
\hline T (P) & $2.018(0.045)$ & $2.051(0.042)$ & $4.345(0.000)$ & $3.881(0.000)$ \\
\hline Duration of infertility (years): & & & & \\
1 - 3 & $4.094 \pm 2.186$ & $11.447 \pm 5.060$ & $7.435 \pm 1.340$ & $9.259 \pm 3.259$ \\
3 - 6 & $4.871 \pm 3.248$ & $11.929 \pm 4.849$ & $8.086 \pm 1.294$ & $10.486 \pm 3.434$ \\
6 or more & $4.822 \pm 2.674$ & $10.978 \pm 5.570$ & $8.467 \pm 0.894$ & $9.156 \pm 3.126$ \\
\hline F(P) & $1.921(0.149)$ & $0.486(0.616)$ & $11.502(0.000)$ & $3.360(0.037)$ \\
\hline Cause of infertility & & & & \\
- Female cause & $4.649 \pm 2.816$ & $11.343 \pm 5.188$ & $7.672 \pm 1.386$ & $8.791 \pm 3.279$ \\
- Male cause & $4.000 \pm 1.677$ & $10.697 \pm 4.908$ & $8.030 \pm 1.104$ & $11.758 \pm 2.538$ \\
- Male \& female cause & $3.885 \pm 2.930$ & $13.923 \pm 4.931$ & $8.885 \pm 0.326$ & $11.923 \pm 2.607$ \\
- Unexplained cause & $7.143 \pm 2.673$ & $9.571 \pm 0.535$ & $7.857 \pm 1.069$ & $8.143 \pm 1.069$ \\
\hline F(P) & $3.252(0.023)$ & $2.677(0.048)$ & $7.040(0.000)$ & $14.258(0.000)$ \\
\hline
\end{tabular}

$\mathrm{F}(\mathrm{P})$ : ANOVA- test \& $\mathrm{P}$ for ANOVA test $\quad \mathrm{T}(\mathrm{P})$ : T-test \& $\mathrm{P}$ for T-test

*: Significant at $\mathrm{P} \leq 0.05$ 
Table (7) Relationship between studied infertile women's socio-demographic characteristics and their total score of quality of life

\begin{tabular}{|c|c|c|}
\hline \multirow[t]{2}{*}{ Socio-demographic characteristics } & Total quality of life & \multirow[b]{2}{*}{$\mathbf{F}(\mathbf{P}) / \mathbf{T}(\mathbf{P})$} \\
\hline & Mean \& SD & \\
\hline $\begin{array}{l}\text { Age (years): } \\
<20 \\
20-30 \\
30-40 \\
40 \text { or more }\end{array}$ & $\begin{array}{l}50.571 \pm 12.637 \\
61.120 \pm 13.793 \\
60.597 \pm 16.432 \\
55.525 \pm 21.885\end{array}$ & $\begin{array}{l}\mathrm{F}(\mathrm{P}) \\
3.044(0.030)\end{array}$ \\
\hline $\begin{array}{l}\text { Level of education: } \\
\text { Illiterate/read \& write } \\
\text { Primary/ preparatory } \\
\text { Secondary } \\
\text { University }\end{array}$ & $\begin{array}{l}58.699 \pm 15.324 \\
52.320 \pm 16.119 \\
59.908 \pm 13.578 \\
72.150 \pm 13.868\end{array}$ & $\begin{array}{l}\mathrm{F}(\mathrm{P}) \\
9.905(0.000)\end{array}$ \\
\hline $\begin{array}{l}\text { Occupation: } \\
\text { Housewife } \\
\text { Working } \\
\end{array}$ & $\begin{array}{l}58.442 \pm 15.465 \\
67.770 \pm 16.602 \\
\end{array}$ & $\begin{array}{l}\mathrm{T}(\mathrm{P}) \\
2.400(0.025)\end{array}$ \\
\hline $\begin{array}{l}\text { Current residence: } \\
\text { Rural } \\
\text { Urban }\end{array}$ & $\begin{array}{l}56.035 \pm 16.077 \\
65.186 \pm 13.522 \\
\end{array}$ & $\begin{array}{l}\mathrm{T}(\mathrm{P}) \\
4.295(0.000)\end{array}$ \\
\hline $\begin{array}{l}\text { Type of family: } \\
\text { Nuclear } \\
\text { Extended }\end{array}$ & $\begin{array}{l}61.238 \pm 14.846 \\
57.003 \pm 16.701 \\
\end{array}$ & $\begin{array}{l}\mathrm{T}(\mathrm{P}) \\
1.868(0.063)\end{array}$ \\
\hline $\begin{array}{l}\text { Family income: } \\
\text { Enough \&can save from it } \\
\text { Only enough } \\
\text { Not enough }\end{array}$ & $\begin{array}{l}68.400 \pm 11.283 \\
61.460 \pm 16.192 \\
53.414 \pm 13.503 \\
\end{array}$ & $\begin{array}{l}F(P) \\
6.586(0.002)\end{array}$ \\
\hline $\begin{array}{l}\text { Age at marriage (years): } \\
<20 \\
20-30 \\
30 \text { or more }\end{array}$ & $\begin{array}{l}60.196 \pm 16.757 \\
58.796 \pm 16.535 \\
59.676 \pm 8.532\end{array}$ & $\begin{array}{l}F(P) \\
0.166(0.847)\end{array}$ \\
\hline $\begin{array}{l}\text { Duration of marriage (years): } \\
1-10 \\
10-20\end{array}$ & $\begin{array}{l}60.032 \pm 15.853 \\
57.296 \pm 15.568\end{array}$ & $\begin{array}{l}\mathrm{T}(\mathrm{P}) \\
1.057(0.294)\end{array}$ \\
\hline
\end{tabular}

$\mathrm{F}(\mathrm{P})$ : ANOVA- test \& $\mathrm{P}$ for ANOVA test

$\mathrm{T}(\mathrm{P})$ : T-test \& $\mathrm{P}$ for T-test

*: Significant at $\mathrm{P} \leq 0.05$

Table (8) Relationship between studied infertile women's infertility history and their total score of quality of life

\begin{tabular}{|l|l|l|}
\hline \multirow{2}{*}{ Infertility history } & Total quality of life & \multirow{2}{*}{ F(P) / T (P) } \\
\cline { 2 - 2 } & Mean \& SD & \\
\hline Type of infertility: & & $\mathrm{T}(\mathrm{P})$ \\
- Primary infertility & $57.215 \pm 15.252$ & $2.819(0.006)$ \\
- Secondary infertility & $63.966 \pm 16.049$ & \\
\hline Duration of infertility (years): & & \\
1 - 3 & $60.225 \pm 15.979$ & $\mathrm{~F}(\mathrm{P})$ \\
3 - 6 & $58.149 \pm 15.995$ & $0.340(0.712)$ \\
6 or more & $59.678 \pm 15.333$ & $\mathrm{~F}(\mathrm{P})$ \\
\hline Cause of Infertility & $54.831 \pm 15.771$ & $14.409(0.000)$ \\
- Female cause & $70.657 \pm 9.099$ & \\
- Male cause & $68.112 \pm 13.029$ & \\
- Both (male \& female) cause & $60.729 \pm 10.637$ & \\
- Unexplained & & \\
\hline
\end{tabular}

$\mathrm{F}(\mathrm{P})$ : ANOVA- test \& $\mathrm{P}$ for ANOVA test

$\mathrm{T}(\mathrm{P})$ : T-test \& $\mathrm{P}$ for T-test

*: Significant at $\mathrm{P} \leq 0.05$ 
Table (9): Correlation between coping strategies and total score of quality of life of studied infertile women

\begin{tabular}{|l|l|l|l|l|l|}
\hline & $\begin{array}{l}\text { Active-avoidance } \\
\text { coping }\end{array}$ & $\begin{array}{l}\text { Active- } \\
\text { confronting } \\
\text { coping }\end{array}$ & $\begin{array}{l}\text { Passive-avoidance } \\
\text { coping }\end{array}$ & $\begin{array}{l}\text { Meaning-based } \\
\text { coping }\end{array}$ & $\begin{array}{l}\text { Total quality } \\
\text { of life }\end{array}$ \\
\cline { 2 - 5 } & $\mathbf{r}(\mathbf{p})$ & $\mathbf{r}(\mathbf{p})$ & $\mathbf{r}(\mathbf{p})$ & $\mathbf{r}(\mathbf{p})$ & $\mathbf{r}(\mathbf{p})$ \\
\hline $\begin{array}{l}\text { Active-avoidance } \\
\text { coping }\end{array}$ & 1 & $\begin{array}{l}-0.524^{*} \\
(0.000)\end{array}$ & $\begin{array}{l}-0.180^{*} \\
(0.011)\end{array}$ & $\begin{array}{l}-0.168^{*} \\
(0.018)\end{array}$ \\
\hline $\begin{array}{l}\text { Active- } \\
\text { confronting } \\
\text { coping }\end{array}$ & $-0.524^{*}$ & 1 & $0.195^{*}$ & $(0.00)$ \\
\hline $\begin{array}{l}\text { Passive-avoidance } \\
\text { coping }\end{array}$ & $(0.000)$ & $-0.180^{*}$ & $(0.005)$ & -0.072 & $0.205^{*}$ \\
\hline $\begin{array}{l}\text { Meaning-based } \\
\text { coping }\end{array}$ & $(0.011)$ & $\begin{array}{l}0.198^{*} \\
(0.005)\end{array}$ & 1 & $0.310)$ & $(0.004)$ \\
\hline $\begin{array}{l}\text { Total quality of } \\
\text { life }\end{array}$ & $-0.168^{*}$ & -0.072 & $0.320^{*}$ & $(0.000)$ & $0.317^{*}$ \\
$(0.018)$ & $(0.310)$ & $(0.000)$ & 1 & $0.000)$ \\
\hline
\end{tabular}

$\mathrm{r}$ (P) Pearson correlation test \& $\mathrm{P}$ for $\mathrm{r}$ test * Correlation is significant at the $\mathrm{P} \leq 0.05$ level (2-tailed)

\section{Discussion}

Despite the evidences of negative impacts of infertility on the couples, more attention is usually paid by the attending health care provider to investigations and treatment rather the complete care of the patients. Couples can manage the infertility crisis by using coping strategies during the diagnosis and treatment of infertility. Coping with infertility during diagnosis and treatment positively affects the health status of the couples involved. ${ }^{(25,26)}$ Since infertility has been known to adversely affect the mental and social health of infertile couples, the assessment of quality of life has become as important as the treatment of infertility. ${ }^{(27,28)}$ Quality of life evaluation allows the understanding of the impact of health conditions or interventions on the patient from a broad perspective; not only the morbidity and mortality, but also the emotional symptoms. Infertility has been shown to impact negatively on the quality of life of women affected by it. ${ }^{(29,30)}$ Coping with infertility is important in order to maintain and/or improve the patient's quality of life. Therefore, this study aimed to assess coping strategies and quality of life among infertile women.

The present study revealed that the infertile women used passive avoidance coping more frequently than other forms of coping strategies (such as active-avoidance, active-confronting or meaning-based coping), while they used active-avoidance coping less frequently than other forms of coping strategies (table 3 ). The infertile women who used passive-avoidance coping hoped for things such as a miracle or felt that the only thing they could do was wait. As well as they have fantasies and wishes about how things might turn out. This finding may be due to that the infertile women in this study perceived less control over their infertility condition. Furthermore, Egyptian culture considers infertility as a social stigma and infertile women try to avoid the condition.

This present study result is supported by Aflakseir A \& Zarei M (2013) ${ }^{(31)}$ and Pottinger A et al (2006) ${ }^{(32)}$ studies. The first revealed that the infertile women had highest score on passive-avoidance coping and the lowest score on active-avoidance. The second revealed that the hoping for a miracle and fantasizing about the outcome were the strategies most commonly used by more than $75 \%$ of infertile women (passive-avoidance coping). It also revealed that the least reported strategy was 'avoiding being around pregnant women or children (active-avoidance coping).

This result is partially similar to the results of at least three other researches. First, Yilmaz T and Oskay $\mathrm{U}(2016){ }^{(33)}$ who found that the infertile women had highest score on passive-avoidance coping and the lowest score on meaning-based coping. Second, Peterson B et al $(2011)^{(34)}$ who found that the infertile women had highest mean score on active-confronting coping (16.1 \pm 3.7$)$, while the lowest mean score on active-avoidance coping (5.8 \pm 1.9$)$. Third, Peterson B et al $(2008){ }^{(35)}$ who reported that the infertile women had highest mean score on active-confronting coping $(16.06 \pm 3.67)$, while the lowest mean score on active-avoidance coping $(6.98 \pm 2.27)$. On the other hand, the present result contradicts Yazdani F et al (2016) ${ }^{(36)}$ in Iran who reported that the infertile women used avoidance coping strategy less than other coping strategies in facing infertility problems.

The results of the current study revealed that there are significant differences between level of education and coping strategies, where the infertile women with higher educational level used activeconfronting coping strategy and meaning-based coping strategy significantly more than the women with low educational level. While the infertile women with low educational level used active and passive-avoidance coping strategies significantly more than the women with higher educational level (table 5). This finding may be attributed to the fact that highly educated women are more prone to use problem-focused approaches, such as doing something regarding a problem, outlining the cause, thinking through the situation and looking for 
information. In higher educated women, relatively high levels of intellectual function are related to positive health-related behaviors and can result in assuming more adaptive coping strategies.

A similar result was observed in Roohafza $\mathrm{H}$ et al $(2009){ }^{(37)}$ research, they did investigate the association of socioeconomic status and life-style factors with coping strategies in Isfahan Healthy Heart Program, Iran. They found that there was a positive relation between high education level and adaptive coping strategies and a negative relation between low education level and mal-adaptive coping strategies.

On the other hand, the same result contradicts to the results of at least three other researches. First, Vinitha D et al $(2015)^{(38)}$ who suggested that there is no significant difference between coping strategies used by the infertile women and their level of education. Second, Lykeridou K et al (2011) ${ }^{(39)}$ who reported that women of low education used higher levels of active-confronting coping than women of high education $(\mathrm{F}=7.997, \mathrm{P}<$ 0.001). They also added that women of low education used passive avoidance coping more than women of high education. However, this difference was marginally statistically significant $(\mathrm{P}=0.051)$. Third, Schmidt et al $(2005)^{(21)}$ who observed that the women from higher social class and level of education used significantly less active-confronting coping and less meaning-based coping when compared with the women from the lowest social class and level of education. Schmidt et al rationalize their findings by the fact that their measure of active-confronting coping strategy included items about seeking infertility-related information. They added that women of higher education had a lower score of active-confronting coping because they might be more informed before starting infertility treatment. ${ }^{(21)}$

The present study results revealed that there was statistically significant differences between age at marriage, type of infertility, causes of infertility and the four coping strategies, while there was statistically significant differences between age, occupation, residence, duration of infertility and two coping strategies namely passive-avoidance and meaning-based coping. On the other hand, there were no significant differences between types of family, family income, duration of marriage coping strategies (table $5 \& 6$ ). In this respect, Vinitha D et al (2015) ${ }^{(38)}$ suggested that there is a significant difference in coping strategies used by the infertile women with type of family $(\mathrm{P}=0.01)$. They add no significant difference was found with other variables such as age, education, occupation, religion, family income and duration of infertility in years. Lykeridou K (2011) ${ }^{(39)}$ also reported that there was no significance association between age, medical characteristics and coping strategies.

The present study results revealed that the infertility reduces quality of life, where the infertile women had lower total mean score of quality of life $59.375 \pm 15.790$, with higher relational subscale and lowest environment subscales (table 4). This is expected since infertile women are more likely to be under pressure from their husbands and relatives. They may also have fears of separation from their husbands or their husbands marrying other wives. This reflects in the poorer scores for these women due to the premium that is placed on childbearing relationship in this part of the world. This explanation supported by two studies which stated that infertility and its treatments have a significant negative impact on a person's quality of life. It has many psychological effects as depression, anxiety, social isolation and deprivation, marital instability, loss of selfesteem and self-confidence, loss of gender identity, loss of control, and feeling of self-blame and guilt that subsequently lead to low quality of life. ${ }^{(40,41)}$

This present study result is supported by Maroufizadeh S et al $(2017){ }^{(42)}$ who found that the total core quality of life was $62.6 \pm 16.9$, and the total treatment quality of life was $58.4 \pm 12.9$, which indicated that the women had the lowest overall quality of life. In addition, this finding is also in agreement with the results of another study done by Masoumi S et al (2016). ${ }^{(43)}$ They concluded that the fertile women obtained higher mean scores in all dimensions of quality of life compared to the infertile ones. Similar results are found by Xiaoli $\mathrm{S}$ et al (2016) ${ }^{(44)}$ who found that the infertile women had significantly lower overall and comprehensive quality of life scores compared with fertile one. In addition, this result is in accordance with the findings of Esmaeilzadeh $\mathrm{S}$ et al (2015). ${ }^{(45)}$ They found that the fertile and infertile women had worse overall quality of life, where the mean score was $61.2 \pm 10.0 \& 61.8 \pm 9.6$ respectively. There was no significant difference in the mean scores in overall quality of life between the fertile and infertile women. They added that the fertile group had higher chance of higher (better) score in overall score domain of quality of life scale than the one that experienced infertility. Esmaeilzadeh $\mathrm{S}$ et al explained their result by the quality of life in fertile group is considered to have been affected by such factors as difficulties due to child care, such as fatigue and insomnia. ${ }^{(45)}$ Also, Sut $\mathrm{H}$ and Kaplan P (2015) ${ }^{(46)}$ reported that infertility negatively affected the emotional status of women, and it negatively affected the mind-body status according to the core FertiQoL scores. They added that the average total score of quality of life was $66.0 \pm 14.5$. This same present study result agrees with the results of at least three other researches. ${ }^{(47,48,49)}$

On the other hand, this result contradicts the results of at least four other studies: First, Parnian R et al $(2017)^{(50)}$ who found that the infertile women had better quality of life, with total mean score $88.22 \pm 13.75$. They add that there was no statistically significant differences in the total mean score of quality of life between fertile and infertile women. Second, Amiri M et al $(2017)^{(51)}$ who found that fertile women's quality of life score was 
not significantly different from that of the infertile women. Third, Norani et al (2012) ${ }^{(52)}$ who found that, although the fertile women had slightly higher scores in all areas of quality of life, there were no statistically significant differences between the two groups in any of the aspects of quality of life. Fourth, Onat G \& Beji N (2012) ${ }^{(53)}$ who found that the mean score of quality of life of the infertile women was higher than the fertile women, but it was not a statistically significant difference. They concluded that infertility did not have a negative impact on quality of life of infertile women. The difference between the quality of life of women in this study with other studies may be due to cultural, social, and economic differences in other societies, which can affect quality of life of people.

The present study revealed that the younger infertile women aged less than 20 years had significantly lower mean score of total quality of life than the older one (table 7). This was expected since younger infertile women had lesser life experience and lack of development of strategies to cope with the infertility problems compared to older one. Furthermore, older women who had a longer history of infertility might be able to cope with their infertility situations in a better way because they had had more infertility related experiences. This result is similar to the results of Rashidi B et al (2008) ${ }^{(54)}$ they found that younger age was a significant predictor for poorer mental health-related quality of life but not for physical health related quality of life. On the contrary, the study of Aduloju O et al (2015) ${ }^{(55)}$ in Ado-Ekiti, Nigeria revealed that the younger age group obtained the lowest scores in all four domains of quality of life, but no significant difference was found. Also, the study of Karabulut A et al (2013) ${ }^{(56)}$ in Denizli, Turkey revealed that the age group itself did not result in differences except in the relational domain of the core subscale, and the environment domain of treatment subscale. Although younger women feel the effect of infertility in their relationship more intensely (relational domain), a younger age did not create any difference in overall quality of life.

In this respect, the present study revealed that the highly educated infertile women had significantly higher mean score of total quality of life compared to low educated one. This finding is in accordance with Rashidi B et al (2008) ${ }^{(54)}$ who found that lower educational level was a significant predictor of the poorer health-related quality of life, both physical and mental health. This may be due to that highly educated infertile women would feel less stigmatized as compared to those with low education. Unlikely, Aduloju O et al (2015) ${ }^{(55)}$ and Karabulut $\mathrm{A}$ et al $(2013)^{(56)}$ reported that the women with primary infertility and higher education had lower total score of quality of life.

The present study revealed that the working infertile women had significantly higher mean score of total quality of life compared to housewives one. A similar result was observed in Aduloju O et al (2015) ${ }^{(55)}$ research, they reported that unemployed infertile women and their husbands had significantly lower total scores of quality of life. The current finding also agrees with that of Heredia et al $(2013)^{(18)}$ who reported lower quality of life for infertile women who are unemployed. This may be due to the demand of the jobs in women who are working could make them to disregard or not to remember their infertility problems at such times, and thus implies a better quality of life. Employment status also reflects on the family income and when both women and husbands are employed, it means a higher family income and better quality of life. ${ }^{(18)}$

The present study results revealed that the infertile women who live in urban areas had significantly higher mean score of total quality of life compared to those who live in rural areas. The finding of the present study is in accordance with Dong Y \& Zhou F (2016) study which revealed that infertile couples resided in rural areas had considerably lower quality of life scores than those from urban residents. ${ }^{(57)}$

The present study results revealed that the women with primary infertility had significantly lower mean of total score of quality of life than those with secondary infertility (table 8). This is expected since being a mother or even being pregnant increases the social respectability of the woman. Therefore, already having a baby probably decreases infertility related problems and leads to an increase in total quality of life score. On the other hand, childless brings about social isolation and decreased tolerability to treatment in women with primary infertility. This result is in agreement with Aduloju $\mathrm{O}$ et al (2015) ${ }^{(55)}$ and Karabulut A et al (2013) ${ }^{(56)}$ researches. They reported that primary infertility has a negative impact on total quality of life scores.

The present study results revealed that no statistically significant difference between the duration of infertility and the total score of quality of life (table 8). This finding is in accordance with Rashidi B et al (2008) ${ }^{(54)}$ who found that the duration of infertility was not significant predictors of poorer health-related quality of life. In contrast, Aduloju O et al (2015) ${ }^{(55)}$ and Karabulut A et al (2013) ${ }^{(56)}$ found that total quality of life was shown to decrease with prolonged duration of infertility. Also Dillu R et al (2013) ${ }^{(58)}$ suggested that the level of quality of life of female partners is dependent upon the duration of infertility.

The present study results revealed that the women with female cause of infertility had significantly lower total score of quality of life than those with other infertility causes (table 8). This may be due to the fact that the women with female infertility causes are blamed (or sometimes they take the blame) more frequently for the couple's infertility and thus, the stigma associated with such blaming (regardless of the diagnosis) causes more distress and deteriorations in quality of life in infertile women. This present study result is supported by 
Parnian R et al (2017) ${ }^{(50)}$ study. They reported that there was a negative and significant correlation between quality of life and the female cause of infertility. On the other hand, Rashidi B et al (2008) ${ }^{(54)}$ found that healthrelated quality of life was better in couples with male factor infertility or both male and female infertility factor. However, the multivariate analysis showed that causes of infertility was not significant predictors of poorer health-related quality of life

The present study results revealed that the total quality of life was found to be positively significant correlated with active-confronting coping, passive-avoidance coping and meaning-based coping, while it was found to be negatively significant correlated with active-avoidance coping (table 9). This may be attributed to that coping has been found to be an important factor closely associated with quality of life. Effective coping strategies play an essential role in maintaining one's physical and psychological wellbeing when dealing with infertility stressors. Effective coping helps to lessen stress, resolve uncomfortable feelings, preserve ability to effectively function in relationships and maintain a positive self-concept that promotes good quality of life. ${ }^{(59)}$

\section{Conclusion}

Based on the findings of this study, it can be concluded that the infertile women used passive avoidance coping more frequently than other forms of coping strategies (such as active-avoidance coping, active-confronting coping or meaning-based coping), while they used active-avoidance coping less frequently than other forms of coping strategies. Moreover, the infertile women obtained lower total mean score of quality of life, this indicate that infertility reduces quality of life. In addition, the total quality of life was found to be positively significant correlated with active-confronting coping, passive-avoidance coping and meaning-based coping, while it was found to be negatively significant correlated with active-avoidance coping.

\section{Recommendation}

\section{Based on the findings of the present study, the following recommendations are suggested:}

1. Psych-educational programs to educate the infertile women and their families about adaptive and maladaptive way of coping with infertility problems.

2. Educational programs should be carried out for nurses to provide them with essential information regarding infertility coping strategies and counseling infertile women.

3. Nurses should be assessed the coping strategies used by infertile women and educate them to promote the use of appropriate coping strategies to enhance quality of life.

4. Further researches should be done to comparative quality of life in infertile and fertile women

\section{References}

[1]. 1. Zegers-Hochschild F, Adamson G, De Mouzon J, Ishihara O, Mansour R, Nygren K, Sullivan E, Poel S, on behalf of ICMART and WHO. The International Committee for Monitoring Assisted Reproductive Technology (ICMART) and the World Health Organization (WHO) Revised Glossary on ART Terminology, 2009. Human Reproduction 2009, 24(11):2683-2687

[2]. Gurunath S, Pandian Z, Anderson R, Bhattacharya S. Defining infertility-a systematic review of prevalence studies. Hum Reprod Update 2011, 17(5):575-588.

[3]. Cooper T, Noonan E, Eckardstein S. "World Health Organization reference values for human semen characteristics". Human Reproductive Update 2010; 16 (3): 231-45.

[4]. Marcia C, Pasquale P. Infertility around the globe: new thinking on gender, reproductive technologies and global movements in the $21^{\text {st }}$ century. Human Reproductive Update 2015; 21 (4): 411-426.

[5]. Community-based Study of the Prevalence of Infertility In Egypt, Egyptian Fertility Care Society (1995). In Inhorn M. Global infertility and the globalization of new reproductive technologies: illustrations from Egypt. SocSci Med 2003; 56: 1937-1951.

[6]. Joshi H, Singh R, Bindu. Psychological distress, coping and subjective well-being among infertile women. J Indian AcadAppl Psychol. 2009; 35(2): 329-336.

[7]. Tovliat V, Motaghedifard M, Tamannaeifar M. A Comparison of Stress Reactions, Coping Styles, Subjective Well-Being and Its Sub-Scales in Fertile and Infertile Women. Journal of Advanced Medical Sciences and Applied Technologies (JAMSAT) 2015; 1(2): 93-97.

[8]. Ghazanfari F, kadampoor E. Relationship between mental health and coping strategies in citizenship of Khoramabad city. J Fundamentals Mental Health 2008; 37: 47-54.

[9]. Peterson B, Newton C, Rosen K, Skaggs G. Gender differences in how men and women who are referred for IVF cope with infertility stress. Human Reproduction 2006; 21(9): 2443-2449.

[10]. Bakhshayesh A, Kazeraninejad M, Mongabadi M, Raghebian M. A comparison of general health and coping strategies in fertile and infertile women in Yazd. Iran J Reprod Med 2012; 10(6): 601-606.

[11]. Jafarzadeh F, Golzari M, Jomehri F, Poursamar S, Sahraian K. The Comparison of Coping Strategies With Stress and Marital Satisfaction in Women on the Basis of Infertility Factor. Women's Health Bull. 2015; 2(2): e25227.

[12]. Chachamovich J, Chachamovich E, Fleck M, Cordova F, Knauth D, Passos E. Congruence of quality of life among infertile men and women: finding from a couple-based study. Hum Reprod. 2009; 24(9): 2151-2157.

[13]. Sexty R, Hamadneh J, Rösner S, Strowitzki T, Ditzen B, Toth B, Wischmann T. Cross-cultural comparison of fertility specific quality of life in German, Hungarian and Jordanian couples attending a fertility center. Health and Quality of Life Outcomes 2016; 14(27):1-8.

[14]. The World Health Organization Quality of Life assessment (WHOQOL): position paper from the World Health Organization SocSci Med. 1995; 41(10):1403-1409. 
[15]. Wiklund I. Assessment of patient-reported outcomes in clinical trials: The example of health-related quality of life. Fundam Clin Pharmacol 2004; 18(3):351-363.

[16]. Chachamovich J, Chachamovich E, Zachia S, Knauth D, Passos E. What variables predict generic and health-related quality of life in a sample of Brazilian women experiencing infertility? Hum Reprod 2007; 22(7):1946-1952.

[17]. Nilforooshan, P, Latifi, Z, Abedi R, Ahmadi A. Quality of life and its different domains in fertile and infertile women. Research of behavioral Science 2008; 4(1/2): 66-70.

[18]. Heredia M, Tenías J, Rocio R, Amparo F, Calleja M, Valenzuela J. Quality of life and predictive factors in patients undergoing assisted reproduction techniques. Eur J Obstet Gynecol Reprod Biol 2013; 167(2):176-180.

[19]. Khayata G, Rizk D, Hasan M, Ghazal-Aswad S, Asaad M. Factors influencing the quality of life of infertile women in United Arab Emirates. Int J Gynaecol Obstet. 2003; 80(2):183-188.

[20]. Yaghmaee F, Mohammadi S, AlaviMjd H. Design and psychometric "Infertile Couples Quality of Life Questionnaire". Fertility and sterility journal 2009; 137-143.

[21]. Schmidt L, Christensen U, Holstein B. The social epidemiology of coping with infertility. Human Reproduction 2005; 20(4): 10441052 .

[22]. Schmidt L. Infertility and assisted reproduction in Denmark. Epidemiology and psychosocial consequences. Thesis for Degree of Doctor of Medical Sciences, University of Copenhagen, Denmark; 2006.

[23]. Boivin J, Takefman J, Braverman A. The Fertility Quality of Life (FertiQoL) tool: development and general psychometric properties. Fertility and Sterility 2011; 96(2): 409-15.

[24]. Boivin, J, Takefman, J, Braverman, A. Development and preliminary validation of the fertility quality of life (FertiQoL) tool. Human Reproduction 2011; 26(8): 2084-2091.

[25]. Sharma C, Subedi D, Rai L, Uprety K. Stress and coping mechanism of infertile women attending infertility clinic Kathmandu. Journal of Universal College of Medical Sciences 2014;2(4):24-28.

[26]. Cunha M, Galhardo A, Pinto-Gouveia J. Experiential avoidance, self-compassion, self-judgment and coping styles in infertility. Sexual \& Reproductive Healthcare 2016; 10(4): 1-7.

[27]. Abedi G, Darvari S, Nadighara A, Rostami F. The Relationship between Quality of Life and Marriage Satisfaction in Infertile Couples Using Path Analysis. J Mazandaran Univ Med Sci 2014; 24(117): 184-193.

[28]. Ferreira M, Vicente S, Duarte J, Chaves C. Quality of Life of Women with Infertility. Procedia - Social and Behavioral Sciences 2015; 165: $21-29$

[29]. Direkvand-Moghadam A, Delpisheh A, Direkvand-Moghadam A. Effect of Infertility on the Quality of Life, a Cross- Sectional Study. Journal of Clinical and Diagnostic Research 2014; 8(10): OC13-OC15

[30]. Moghadam A, Delpisheh A, Montazeri A, Sayehmiri K. Quality of Life in Infertile Menopausal Women; Development and Psychometric of an Instrument. Journal of Clinical and Diagnostic Research 2016;10(6): IC01-IC05

[31]. Aflakseir A, Zarei M. Association between Coping Strategies and Infertility Stress among a Group of Women with Fertility Problem in Shiraz, Iran. J ReprodInfertil. 2013; 14(4):202-206.

[32]. Pottinger A, McKenzie C, Fredericks J, DaCosta V, Wynter S, Everett D, Walters Y. Gender Differences in Coping with Infertility among Couples Undergoing Counseling for In Vitro Fertilization Treatment. West Indian Med J 2006; 55 (4): 237-242.

[33]. Yilmaz T and Oskay U. The Copenhagen Multi-centre Psychosocial Infertility (COMPI) Fertility Problem Stress and Coping Strategy Scales: A Psychometric Validation Study in Turkish Infertile Couples. International Journal of Caring Sciences 2016; 9(2): 452- 462 .

[34]. Peterson B, Pirritano M, Block J, Schmidt L. Marital benefit and coping strategies in men and women undergoing unsuccessful fertility treatments over a 5-year period. Fertility and Sterility 2011; 95(5): 195-1963.

[35]. Peterson B, Pirritano M, Christensen U, Schmidt L. The impact of partner coping in couples experiencing infertility. Human Reproduction 2008; 23(5): 1128-1137.

[36]. Yazdani F, Kazemi A, Ureizi-Samani H. Studying the Relationship between the Attitude to Infertility and Coping Strategies in Couples Undergoing Assisted Reproductive Treatments. J Reprod Infertil. 2016; 17(1):56-60.

[37]. Roohafza H, Sadeghi M, Shirani S, Bahonar A, Mackie M, Sarafzadegan N. Association of Socioeconomic Status and Life-style Factors with Coping Strategies in Isfahan Healthy Heart Program, Iran. Croat Med J. 2009; 50: 380-6

[38]. Vinitha D, Angelita N, Shobha K. Psychosocial wellbeing and coping strategies of infertile women seeking infertility treatment. Journal of Infertility and Reproductive Biology 2015; 3(2): 174-178.

[39]. Lykeridou K, Gourounti K, Sarantaki A, Loutradis D, Vaslamatzis G, Deltsidou A. Occupational social class, coping responses and infertility-related stress of women undergoing infertility treatment. Journal of Clinical Nursing 2011; 20: 1971-1980

[40]. Chachamovich JR, Chachamovich E, Ezer H, Fleck MP, Knauth D, Passos EP. Investigating quality of life and health-related quality of life in infertility: a systematic review. J PsychosomObstetGynaecol. 2010; 31(2): 101-110.

[41]. Maroufizadeh S, Karimi E, Vesali S, Samani R. Anxiety and depression after failure of assisted reproductive treatment among patients experiencing infertility. Int J Gynaecol Obstet. 2015; 130(3): 253-256.

[42]. Maroufizadeh S, Ghaheri A, Amini P, Samani R. Psychometric Properties of The Fertility Quality of Life Instrument in Infertile Iranian Women. International Journal of Fertility and Sterility 2017; 10(4): 371-379.

[43]. Masoumi S, Garousian M, Khani S, Oliaei S, Shayan A. Comparison of Quality of Life, Sexual Satisfaction and Marital Satisfaction between Fertile and Infertile Couples. International Journal of Fertility and Sterility 2016; 10(3): 290-296.

[44]. Xiaoli S, Mei L, Junjun B, Shu D, Zhaolian W, Jin W, Ju Q, Wanli S, Huali Z, Li J, Dong L, Li P, Xiaojin H. Assessing the quality of life of infertile Chinese women: a cross-sectional study. Taiwanese Journal of Obstetrics \& Gynecology 2016; 55: 244e250

[45]. Esmaeilzadeh S, Delavar M, Pasha N. Quality of life of Iranian married women: a comparative study of women who had experienced infertility with fertile ones through Health, Wellness and Quality of Life Questionnaire. Caspian Journal of Reproductive Medicine 2015; 1(1):13-19.

[46]. Sut, $\mathrm{H}$ and Kaplan P. Quality of life in women with infertility via the FertiQoL and the Hospital Anxiety and Depression Scales. Nursing and Health Sciences 2015; 17: 84-89

[47]. Hsu P, Lin M, Hwang J, Lee M, Wu M. The fertility quality of life (FertiQoL) questionnaire in Taiwanese infertile couples. Taiwanese Journal of Obstetrics \& Gynecology 2013; 52: 204e209.

[48]. Valsangkar S, Bodhare T, Bele S. An evaluation of the effect of infertility on marital, sexual satisfaction indices and health -related quality of life in women. Journal of Human Reproductive Science 2011; 4(2):80-85.

[49]. Hassanin I, Abd-El-Raheem T, Shahin A. Primary infertility and health-related quality of life in Upper Egypt. International Journal of Gynecology \& Obstetrics 2010; 110(2): 118-121.

[50]. Parnian R, Farzad Poorgholami1, Nehle Parandavar2, Safeih Jamali2 \&Fatemeh Shakeri3. A Comparative Study of Quality of Life in Infertile and Fertile Women Referred to Jahrom Infertility Clinics. Global Journal of Health Science 2017; 9(4): 174-181. 
[51]. Amiri M, Chaman R, Sadeghi Z, Khatibi M, Ranjbar M. Quality of Life Among Fertile and Infertile Women. Iran J Psychiatry Behav Sci. 2017; In Press (In Press):e5641.

[52]. Norani S, Jonaedi E, Shakri M, Mokhber N. A comparative study of quality of life in infertile and fertile women referred to health clinics in Mashhad City. Iranian Journal of obstetrics, gynecology and infertility 2012; 15(7): 24-31

[53]. Onat G, Beji N. Effects of infertility on gender differences in marital relationship and quality of life: a case-control study of Turkish couples. European Journal of Obstetrics \& Gynecology and Reproductive Biology 2012; 165(2): 243-248.

[54]. Rashidi B, Montazeri A, Ramezanzadeh F, Shariat M, Abedinia N, Ashrafi M. Health-related quality of life in infertile couples receiving IVF or ICSI treatment. BMC Health Services Research 2008; 8:186

[55]. Aduloju O, Akintayo A, Olofinbiyi B, Awoleke J, Ade-Ojo I, Dada M. Predictors of quality of life among infertile women in a south-western nigerian teaching hospital. International Journal of Tropical Medicine and Public Health 2015; 5(1): 8-12.

[56]. Karabulut A, Özkan S, Oguz N. Predictors of fertility quality of life (FertiQoL) in infertile women: Analysis of confounding factors. Eur J Obstet Gynecol Reprod Biol 2013; 170: 193-7.

[57]. Dong Y, Zhou F. Comparison of fertility quality of life between urban and rural infertile couples. Int J Clin Exp Med 2016; 9(5):8664-8670

[58]. Dillu R, Sheoran P, Sarin J. An Exploratory Study to Assess the Quality of Life of Infertile Couples at Selected Infertility Clinics in Haryana. IOSR Journal of Nursing and Health Science 2013; 2(3): 45-51

[59]. Kolahi L, Asemi N, Mirzaei M, Adibi N, Beiraghdar M, Mehr A. The relationship between quality of life and coping strategies in polycystic ovary syndrome patients. Advanced Biomedical Research | 2015; 4:168 